(C) [2009] IEEE. Reprinted, with permission, from Huang, Yunkai; Lin, Heyun; Zhu, Jianguo; Guo, Youguang. 2009, 'Magneto-thermal analysis of a high-speed SMC motor based on 3D FEA', Proceedings of the 8th International Conference on Power Electronics and Drive Systems (PEDS), pp. 563-567.This material is posted here with permission of the IEEE. Such permission of the IEEE does not in any way imply IEEE endorsement of any of the University of Technology, Sydney's products or services. Internal or personal use of this material is permitted. However, permission to reprint/republish this material for advertising or promotional purposes or for creating new collective works for resale or redistribution must be obtained from the IEEE by writing to pubs-permissions@ieee.org. By choosing to view this document, you agree to all provisions of the copyright laws protecting it. 


\section{Magneto-Thermal Analysis of a High-Speed SMC Motor Based on 3-D FEA}

\author{
Yunkai Huang, Heyun Lin \\ School of Electrical Engineering \\ Southeast University \\ Nanjing, P. R. China
}

\begin{abstract}
This paper presents a magneto-thermal model for high-speed SMC motor. Due to high frequency, core loss is much greater than other losses and it is of crucial importance to be able to calculate the core loss accurately. By using time-stepping FEA, the total core loss is computed by summing up the separately calculated alternating and rotational hysteresis, eddy current and anomalous losses in each element. The core loss in each element is coupled with the thermal analysis by keeping the same mesh structure between the magnetic and thermal analyses. Other losses such as air frictional loss and rotor loss are included in the model due to high speed. This model is applied to a $2 \mathrm{~kW}, 20 \mathrm{krpm}$ claw pole motor with a SMC core. The 3-D FEA is conducted for the core loss and temperature calculation because flux path in claw pole motor is purely three dimensional. The results are compared with experiments.
\end{abstract}

Keywords- Core losses, finite element analysis, high-speed motors, soft magnetic composite (SMC) materials, thermal analysis.

\section{INTRODUCTION}

High-speed motors are being used in many applications, such as machine tool spindles, aerospace, centrifugal compressors, vacuum pumps, friction welding units, turbine generators, and so on. Due to high operating frequency, core loss is much greater than other losses and is the main source of heat. In a rotating motor, a great percentage of the total core loss is caused by the rotating magnetic field [1], and therefore, the rotational core loss should be accurately calculated. Because motors are rated by the thermal conditions, or the operating temperature, the thermal analysis is a critical procedure in motor design, especially for high speed motors. For thermal analysis, there are two basic methods: the lumped parameter thermal network and the FEM. To get the accurate temperature distribution, the FEM is the only choice because of the distributed heat sources, including both the copper and core losses in each element, whereas to obtain the core loss in each element, the finite element analysis of magnetic field is required, resulting in a coupled magneto-thermal problem.

The coupling between the magnetic and thermal fields can be accomplished by either a direct or sequential coupling. The direct coupling is more advantageous when the coupled field interaction is highly nonlinear and is best solved in a single solution of a group of coupled equations, while the sequential method is more efficient and flexible because the two analyses can be performed independently.

\author{
Jianguo Zhu, Youguang Guo \\ Faculty of Engineering \\ University of Technology, Sydney \\ Sydney, Australia
}

The coupling may be recursive where the iterations between the different physics are performed until the desired level of convergence is achieved. Since the core loss calculation needs complicated post processing after the solution of magnetic field, the sequential coupling method is more appropriate for the coupled magneto-thermal problem in electrical motors.

This paper presents a magneto-thermal model to accurately calculate the temperature distribution in a high speed claw pole PM motor with a SMC core. The 3-D FEA is used for the coupled magnetic and thermal field analyses. As a main source of heat, the rotational core loss in each finite element is calculated and coupled with the thermal analysis by keeping the same mesh structure. The results are compared with experiments.

\section{STRUCTURE AND DIMENSION}

Fig. 1 illustrates the topology of the high speed three phases claw pole PM motor, which has three stator stacks, each stack consisting of the claws, the yoke and the coil. The three stator stacks are shifted for 120 electrical degrees from each other to get a smooth torque. Because of the complex geometry, the magnetic field in a claw pole motor is truly 3-D at any time. On the other hand, the claw pole structure is difficult to manufacture by using the conventional laminated silicon steel. The manufacturing process can be greatly simplified by using the molding technique and hence the SMC material would be very suitable for this structure.

The SMC material produced by the powder metallurgical technology has undergone a significant development in the past few years because of its unique properties and the great potential of low cost motor manufacturing. To explore the full potential of the SMC material, this high speed motor is designed. Table I lists the key dimensions and parameters.

\section{Finite ELEMENT MODEL AND MESH}

The claw pole motor has a structure of 3-D field, and therefore 3-D FEA is required for the accurate calculation of motor parameters, performance and temperature distribution. In this paper, ANSYS, a commercial package, is used. Because of the periodical symmetry, only one pole pitch of the machine, as shown in Fig. 2, needs to be calculated. 


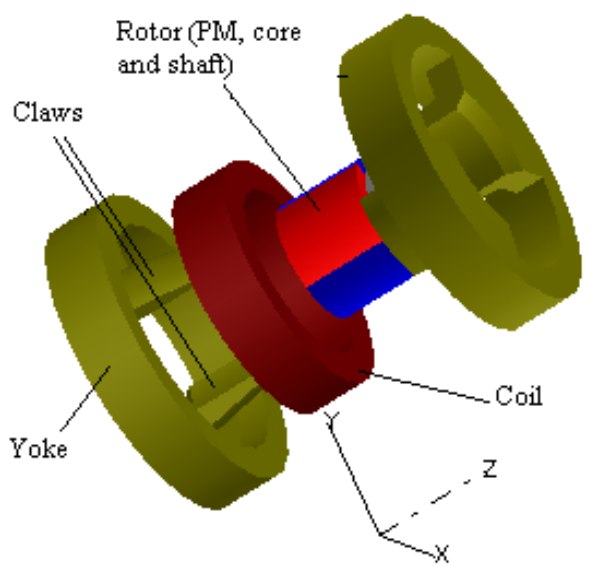

Fig. 1. Magnetically relevant parts of a stack of claw pole motor

TABLE I

Key Dimensions AND Design PaRAmeters

\begin{tabular}{cc}
\hline Dimension & Value \\
\hline Number of phases & 3 \\
Rated power (W) & 2000 \\
Rated frequency (Hz) & 666.7 \\
Rated speed (r/min) & 20000 \\
Number of poles & 4 \\
Stator core material & SOMALOY $^{\mathrm{TM}} 500$ \\
Stator outer diameter (mm) & 78 \\
Rotor core material & SOMALOY \\
Rotor outer diameter $(\mathrm{mm})$ & 29 \\
Rotor inner diameter (mm) & 18 \\
PMs & NdFeB $(\mathrm{N} 35)^{\mathrm{TM}}$ \\
Magnet arc & $75^{\circ}$ \\
Magnet radial length (mm) & 2 \\
Airgap length (mm) & 1 \\
Axial length (mm) & 48 \\
\hline
\end{tabular}

In order to couple the core loss directly with the thermal field analysis, the size and number of elements in thermal field analysis must be kept the same as those in the magnetic filed analysis. The mapped mesh is a preference, and the geometry must be built as a series of fairly regular volumes. Therefore, the stator core in one pitch region is divided into 18 parts, the winding into 3 parts, and the rotor core into 3 parts to use the mapped mesh in both fields. The division lines between these parts can be seen in Fig. 2. The mesh of the FEA model is shown in Fig. 3. The whole solution region is meshed into 5568 hexahedral elements. This is enough to calculate the flux, but more elements are needed when calculating the torque.

\section{HEAT SOURCES}

\section{A. Stator Core Loss}

The core loss calculation is crucial for high speed motors because it is the dominant power loss component due to the high operating frequency. An accurate model including the rotational core loss described in [1] is employed to predict the core loss in the 3-D flux SMC motor. Based on the time-stepping FEA, the total core loss is computed by calculating the hysteresis, eddy current and anomalous losses separately in each element under the alternating and rotational magnetic fields.

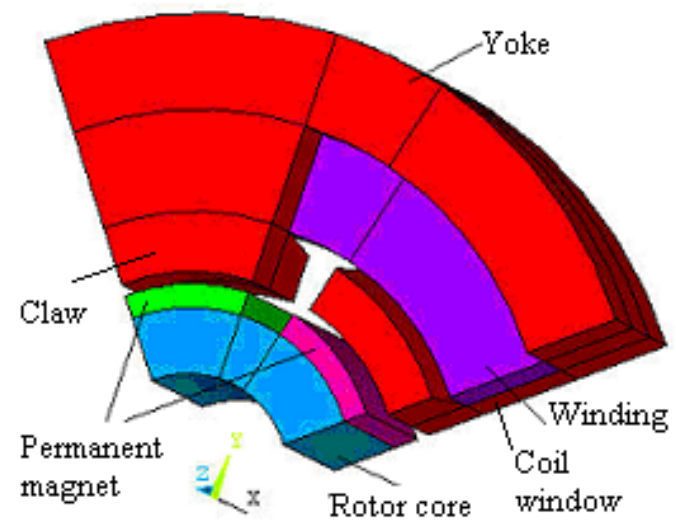

Fig. 2. Region of SMC motor for field solution

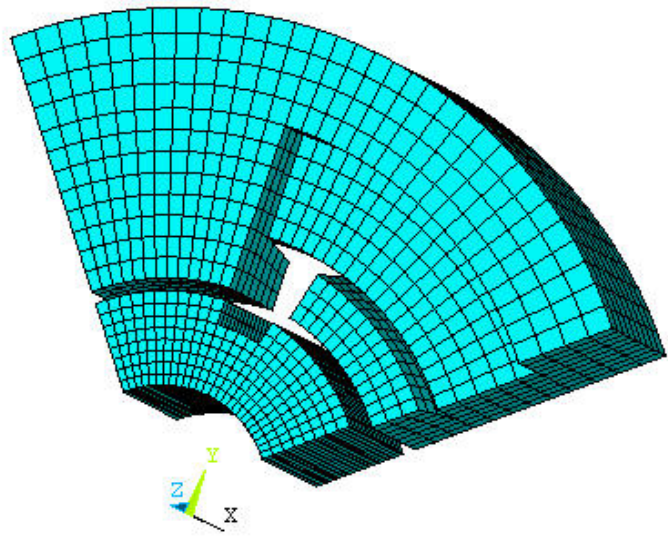

Fig. 3. The mesh of FEA model

If the magnetic flux density is sinusoidal, the alternating core loss is calculated by

$$
P_{a l t}=K_{a h} f B_{m}{ }^{h}+K_{a e}\left(f B_{m}\right)^{2}+K_{a a}\left(f B_{m}\right)^{3 / 2}
$$

and the core loss with circular rotational flux density by:

$$
P_{r o t}=P_{r h}+K_{r e}\left(f B_{m}\right)^{2}+K_{r a}\left(f B_{m}\right)^{3 / 2}
$$

where

$$
\frac{P_{r h}}{f}=a_{1}\left[\frac{1 / s}{\left(a_{2}+1 / s\right)^{2}+a_{3}^{2}}-\frac{1 /(2-s)}{\left[a_{2}+1 /(2-s)\right]^{2}+a_{3}^{2}}\right]
$$

and

$$
s=1-\frac{B_{m}}{B_{s}} \sqrt{1-\frac{1}{a_{2}^{2}+a_{3}^{2}}}
$$

The coefficients $K_{a h}, h, K_{a e}, K_{a a}, K_{r e}, K_{r a}, a_{1}, a_{2}, a_{3}$ and $B_{s}$ can be deduced from the data measured by the rotational core loss tester, and $B_{m}$ is the peak value of sinusoidal flux density. For SOMALOY ${ }^{\mathrm{TM}} 500, K_{a h}=0.1402, h=1.548$, $K_{a e}=1.233 \mathrm{E}-5, K_{a a}=3.645 \mathrm{E}-4, K_{r e}=2.303 \mathrm{E}-4, K_{r a}=0, a_{1}$ $=6.814, a_{2}=1.054, a_{3}=1.445$, and $B_{s}=2.134 \mathrm{~T}$.

The core loss with an elliptical $B$ is predicted from the alternating and circularly rotating core losses by 


$$
P_{f e}=R_{B} P_{\text {rot }}+\left(1-R_{\mathrm{B}}\right)^{2} P_{\text {alt }}
$$

where $R_{B}=B_{\text {min }} / B_{\text {maj }}$ is the axis ratio, and $B_{\text {maj }}$ and $B_{\text {min }}$ are the major and minor axes of the elliptical $\boldsymbol{B}$ locus.

The core loss is computed based on time-stepping FEA. One electrical period is divided into 18 steps. The meshing of stator and rotor is kept same in each step. The core loss in each element is calculated, and then the total core loss of the motor is obtained by summing up the losses of all the elements.

The flux density at the typical element of the yoke is shown in Fig. 4. The distribution of stator core loss at noload (20 krpm) is shown in Fig. 5. The core loss in the claws is greater than that in other parts of stator core because the changing amplitude of magnetic filed is greater than that in other parts.

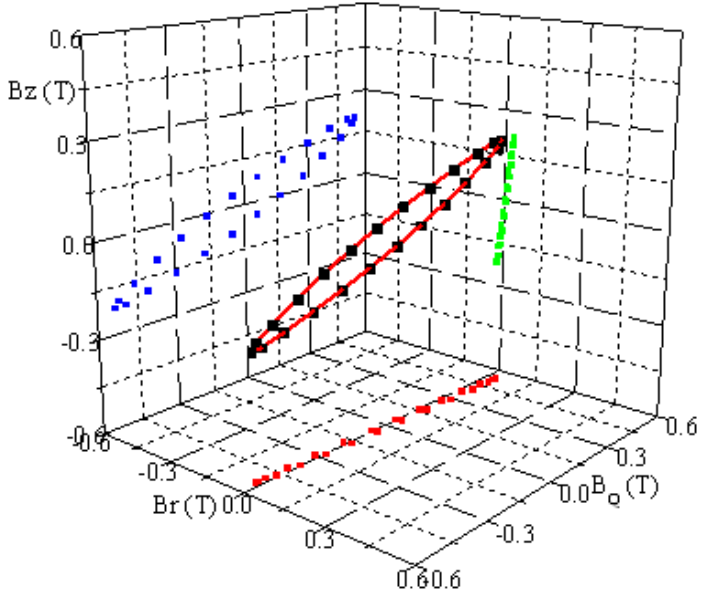

Fig. 4. The flux density locus at typical element of the yoke

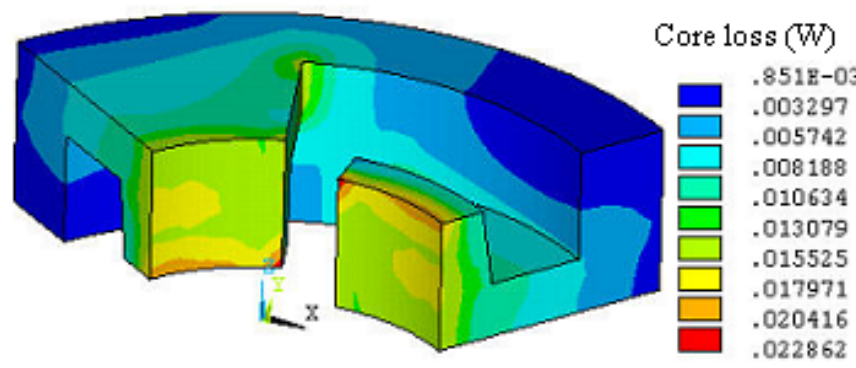

Fig. 5. the distribution of core loss at no-load

\section{B. Stator Winding Losses}

A ring coil is used in the claw pole motor, as shown in Fig. 1. The copper losses consist of $\mathrm{I}^{2} \mathrm{R}$ loss and eddy current loss. Because there is no end winding, the resistance is calculated readily from the dimensions.

The eddy current loss comes form [2]: (a) skin effect resulting from the same source conductors, and (b) proximity effect resulting from the motion of the PM. Since multi-strand winding is used and electrical frequency is low (about $1 \mathrm{kHz}$ ), the skin depth is larger than the radius of the copper wire. Therefore, the skin effect can be ignored. Through the FEA at different rotor positions, it is found that the magnetic density in the winding varies very little, and hence the effects of eddy currents can be safely ignored.
The stator winding loss is calculated by $I^{2} R$, where $R$ is the nominal resistance corrected for temperature.

\section{Air Firiction Losses}

Because ring magnet is assembled on the shaft, the surface of rotor can be assumed to be smooth. The air friction is calculated using a formula found in [3].

$$
P_{a f}=C_{f} \pi \rho \omega^{3} r^{4} l
$$

where $\rho\left[\mathrm{Kg} / \mathrm{m}^{3}\right]$ is the density of the air, $\omega[\mathrm{rad} / \mathrm{s}]$ the angular velocity, $l[\mathrm{~m}]$ the axial length of the rotor and $r$ the radius. The friction coefficient $C_{f}$ is calculated by

$$
\begin{gathered}
C_{f}=\frac{0.0152}{\operatorname{Re}_{\delta}^{0.24}}\left[1+\left(\frac{8}{7}\right)^{2}\left(\frac{4 R e_{a}}{R e_{\delta}}\right)^{2}\right]^{0.38} \\
\operatorname{Re}_{\delta}=\frac{\rho \omega r \delta}{\mu} \\
\operatorname{Re}_{a}=\frac{\rho v_{a} 2 \delta}{\mu}
\end{gathered}
$$

where $\mu$ is the dynamic viscosity of air, $v_{a}[\mathrm{~m} / \mathrm{s}]$ the line speed of the rotor, and $\delta[\mathrm{m}]$ the radial airgap length.

\section{Rotor Loss}

In a high speed PM motor, the rotor loss generated by induced eddy currents in the rotor back iron, the PMs, and the retaining sleeve can be a considerable part of the total loss. Specifically, the rotor eddy current loss can result from: (a) stator slotting, (b) a nonsinusoidal stator MMF distribution, and (c) nonsinusoidal phase current waveforms, which result from six-step commutation and PWM [4].

Analytical or FEA methods used in most literatures to calculate the rotor eddy current loss are based on 2-D magnetic field analysis, and the slotting effect is neglected. Therefore, they cannot be used in claw pole motor design because magnetic flux path is purely 3-D and it has large slot openings. A dynamic circuit model described in [5] is used to calculate the eddy current loss in the PM in this paper. As shown in Fig. 6, the cross section of the PM is divided into a number of slice pairs. Each slice pair forms an assumed eddy current loop. The thickness of a slice $\Delta y$ is chosen such that within the slice the variation of eddy current density is almost linear. Inside the slice, therefore, the reaction of eddy currents on the magnetic field distribution can be ignored, and the flux density can be considered as uniform distribution. The eddy current loss in the $k^{\text {th }}$ slice pair can then be calculated by

$$
P_{e k}=\left(\frac{2 l_{a} l_{r} \Delta y}{\sigma}\right) J_{k}{ }^{2}\left\{\frac{1}{3}\left[1+\left(1+\frac{J_{k-1}}{J_{k}}\right) \frac{J_{k-1}}{J_{k}}\right]\right\}
$$

where $l_{a}$ and $l_{r}$ are the axial and radial lengths of PM, respectively, $\sigma$ is the conductivity of PM, $J_{k}$ and $J_{k-1}$ are the eddy current densities in the $k$ th and $k$ - 1 th slice pair, and

$$
J_{k}=\sigma\left(\frac{d B_{r}}{d t}\right)_{k} \Delta y+J_{k-1}
$$

At 20krpm, the calculated losses are listed in Table II. 


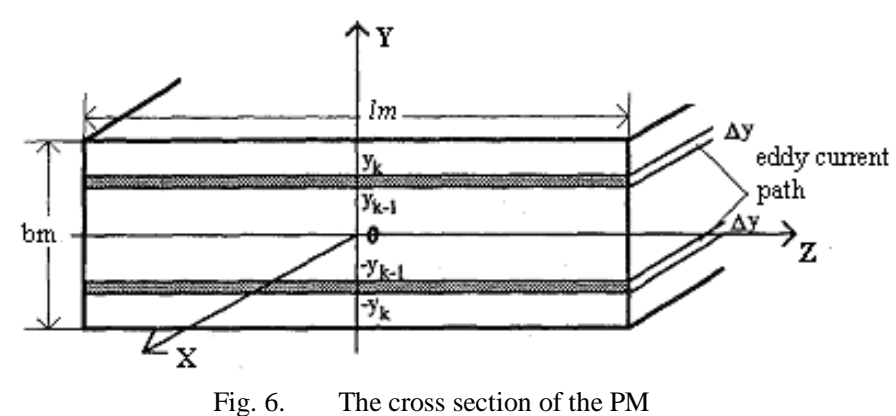

TABLE II

LOSSES AT 20KR/MIN (THREE STACKS)

\begin{tabular}{ccc}
\hline Loss $(\mathbf{W})$ & No-load & Full-load \\
\hline Stator winding & 0 & 16.6 \\
Stator core & 86.1 & 92 \\
Magnet & 10.4 & 10.4 \\
Air friction & 6.2 & 6.2 \\
\hline
\end{tabular}

\section{THERMAL FIELD ANALYSIS}

The partial differential equation of the heat conduction and convection is expressed as

$$
\rho c\left(\frac{\partial T}{\partial t}+\{V\}^{T}\{L\} T\right)=\{L\}^{T}([D]\{L\} T)+Q
$$

where $\rho[\mathrm{Kg} / \mathrm{m} 3]$ is the mass density, $c[\mathrm{~J} /(\mathrm{Kg} * \mathrm{~K})]$ the specific heat, $\mathrm{T}[\mathrm{K}]$ the temperature, $\mathrm{t}[\mathrm{s}]$ the time, $\{L\}$ the vector operator, $\{V\}$ the velocity vector for mass transport of heat, $[D]$ the thermal conductivity matrix, and $Q\left[\mathrm{~W} / \mathrm{m}^{3}\right]$ the heat generation rate per unit volume.

For steady state analysis, the left hand side terms in (10) are zero. The core loss in each element obtained from the magnetic field analysis is applied as the body loads to the corresponding element in the thermal analysis by keeping the same size and number of the element. The Dirichlet type of boundary condition specifies the boundary temperature $T^{*}$, and is applicable to the surface between the frame and outer air. The Neumann type of boundary condition specifies the heat flux flow $q_{n}$ through a boundary, and the Newton's convection boundary condition has the form of

$$
q_{n}=n \bullet(k \nabla T)=\alpha\left(T-T_{a m b}\right)
$$

The heat flux flow through the boundaries to the surrounding is described with the heat transfer coefficient $\alpha$ and the external temperature $T_{a m b}$. The convective heat transfer to the surroundings is dependent on the geometry and the cooling conditions. Air gap convection coefficient is determined by two main quantities: the ruggedness of the rotor and stator surfaces and the peripheral speed of the rotor surface. By assuming a smooth surface, the convection coefficient can be calculated by experiential formula [6]:

$$
\begin{gathered}
h_{\delta}=28\left(1+\omega_{\delta}{ }^{0.5}\right) \\
\omega_{\delta}=\sqrt{\left(0.5 v_{r}\right)^{2}+v_{a}{ }^{2}}
\end{gathered}
$$

where $v_{r}$ and $v_{a}$ are the line and axial speed of the rotor surface, respectively.

The thermal conductivities of different materials are chosen as: $\mathrm{SMC}=20$, air $=0.027$, aluminum $=220$, copper $=380$, steel $=50$, magnet $=9$, glue $=0.5$ and insulation $=0.18$.

The temperature distribution at no-load is shown in Fig. 7. Compared with Fig. 5, it is shown that the temperature is mainly affected by the core loss. The temperatures of the winding and magnets are well below $100^{\circ} \mathrm{C}$, the maximum temperature of the insulating material and magnet.

\section{TEST}

A testing bench, as shown in Fig. 8, is set up to measure the core loss and temperature at no-load generating operation. The core loss at no-load is measured by separating the core loss from the mechanical loss using the dummy rotor method.

The calculation and measurement results of core loss are compared in Fig. 9. Below 12krpm, the error is less than $10 \%$, but the maximum error is about $15 \%$ when the rotate speed over $12 \mathrm{krpm}$. The possible reasons are: (1) loss coefficients derived from the rotational core loss data at low frequency have some errors when they are used in high frequency; and (2) the rotor loss cannot be separated from the measurement.

The temperature is measured by an infrared temperature probe. At $20 \mathrm{krpm}$ no-load, the frame temperature is $331.4 \mathrm{~K}$, and stator yoke $333.5 \mathrm{~K}$. The measured temperature is higher than the calculated since the actual loss is greater than the calculation.

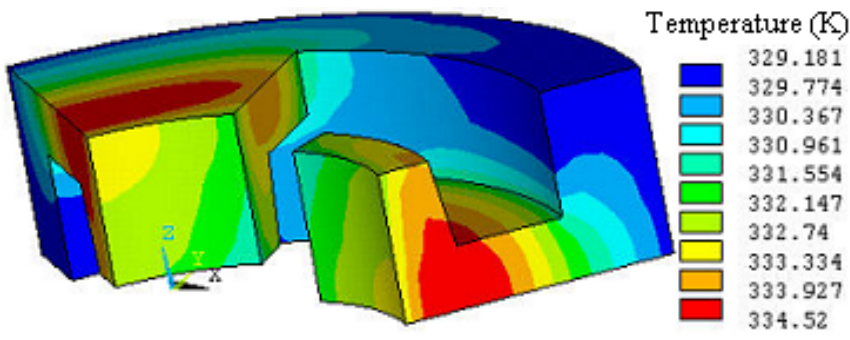

Fig. 7. The temperature distribution of stator core

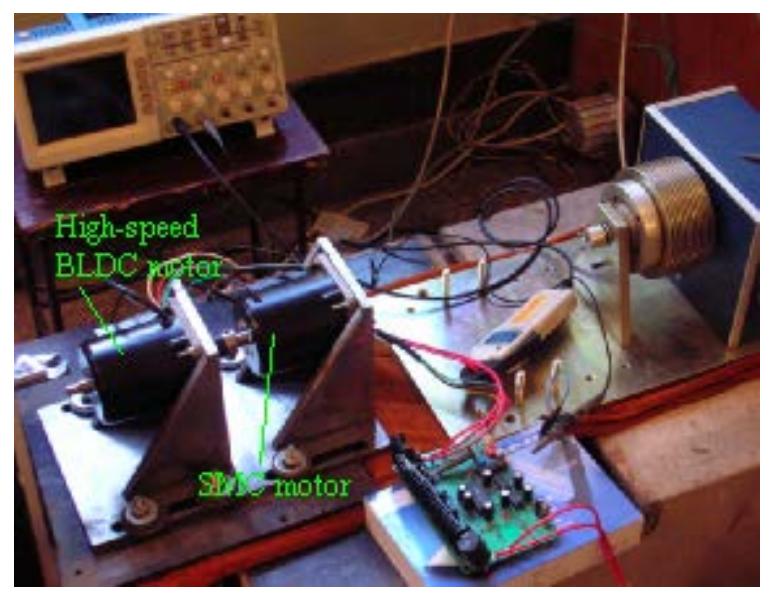

Fig. 8. Test bench for core loss measurement 


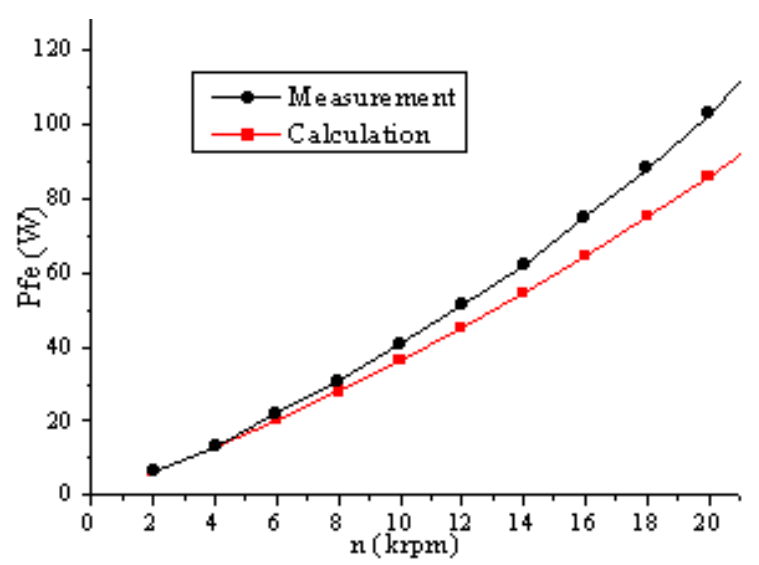

Fig. 9. The calculation and measurement results of core loss

\section{CONCLUSIONS}

This paper presents a coupled magneto-thermal model for accurately calculating temperature distribution inside a motor. The core loss is calculated by the rotational core loss model after solving the 3-D magnetic field, and coupled directly into the 3-D thermal field analysis. The temperature distribution of a high speed claw pole motor with a soft magnetic composite core is calculated as an example. The validity of method is verified by experimental measurements.

\section{REFERENCES}

[1] J. G. Zhu and V. S. Ramsden, "Improved formulations for rotational core losses in rotating electrical machines," IEEE Trans. On Magnetics, vol. 34, pp. 2234-2242, July 1998.

[2] L. Zheng, T. X. Wu, D. Acharya, et al, "Design of a Super-High Speed Permanent Magnet Synchronous Motor for Cryogenic Applications," 2005 IEEE Intern. Conf. on Electric Machines and Drives, pp. 874-881.

[3] J. Saari, "Thermal analysis of high-speed induction machines," in Electrical Engineering, vol. Doctor: Helsinki University of Technology, Finland, 1998, pp. 73.

[4] Z. Q. Zhu, K. Ng, N. Schofield, and D. Howe, "Improved Analytical Modeling of Rotor Eddy Current Loss in Brushless Machines Equipped with Surface-Mounted Permanent Magnets," IEE Proc.Electr. Power Appl., vol. 151, pp. 641-650, 2004.

[5] J. G. Zhu, S. Y. R. Hui, and V. S. Ramsden, "A generalized dynamic circuit model of magnetic cores for low- and highfrequency applications. I. Theoretical calculation of the equivalent core loss resistance," IEEE Transaction on Power Electronics, vol. 11, pp. 246-250, 1996.

[6] Y. T. Wei, D. W. Meng, and J. B. Wen, The heat transfer in electrical machines. Beijing: China mechanism publisher, 1998. 